\title{
CD147 promotes Src-dependent activation of Rac1 signaling through STAT3/DOCK8 during the motility of hepatocellular carcinoma cells
}

\author{
Shi-Jie Wang ${ }^{1, *}$, Hong-Yong Cui ${ }^{1, *}$, Yan-Mei Liu ${ }^{1, *}$, Pu Zhao ${ }^{2}$, Yang Zhang ${ }^{1}$, Zhi- \\ Guang Fu' ${ }^{1}$, Zhi-Nan Chen ${ }^{1}$ and Jian-Li Jiang ${ }^{1}$ \\ ${ }^{1}$ Cell Engineering Research Center \& Department of Cell Biology, State Key Laboratory of Cancer Biology, National Key \\ Discipline of Cell Biology, Fourth Military Medical University, Xi'an, China \\ ${ }^{2}$ College of Life and Health Sciences, Northeastern University, Shenyang, China \\ * These authors contributed equally to this work \\ Correspondence to: Jian-Li Jiang, email: jiangjl@fmmu.edu.cn \\ Zhi-Nan Chen, email: zhinanchen@fmmu.edu.cn \\ Keywords: CD147; SrC; Rac1; DOCK8; STAT3
}

Received: August 28, $2014 \quad$ Accepted: November 15, $2014 \quad$ Published: November 16, 2014

This is an open-access article distributed under the terms of the Creative Commons Attribution License, which permits unrestricted use, distribution, and reproduction in any medium, provided the original author and source are credited.

\section{ABSTRACT}

Metastasis is considered a dynamic process in tumor development that is related to abnormal migration and invasion. Tumor cells can move as individual cells in two interconvertible modes: mesenchymal-type and amoeboid. Previously, we reported that the interaction between CD147 and Annexin II can inhibit the amoeboid movement in hepatocellular carcinoma (HCC) cells. However, the mechanism of CD147 involved in mesenchymal movement is still unclear. Notably, our results show overexpression of CD147 led to mesenchymal-type movement in HCC cells. Evidence indicated that the mesenchymal-type cell movement induced by CD147 was Src dependent, as observed by confocal microscopy and Rac1 activity assay. The phosphorylation of Src (pY416-Src) can be up-regulated by CD147, and this regulation is mediated by focal adhesion kinase (FAK). Next, we identified DOCK8 as a GEF for Rac1, a key molecule driving mesenchymal-type movement. We also found that Src promotes STAT3 phosphorylation and STAT3 facilitates DOCK8 transcription, thus enhancing DOCK8 expression and Rac1 activation. This study provides a novel mechanism of CD147 regulating mesenchymal-type movement in HCC cells.

\section{INTRODUCTION}

The metastatic dissemination of tumors is the main cause of death for most cancer patients [1,2]. Tumor metastasis is a highly complex and multistep process that requires a tumor cell to modulate its ability to adhere, invade into the surrounding extracellular matrix, migrate, and proliferate at a secondary site [3]. Migratory cancer cells undergo dramatic molecular and cellular changes by remodeling their cell-cell, cell-matrix adhesion, actin cytoskeleton, and molecular processes involved in various signaling networks [2, 4, 5]. Two different, interconvertible modes of cell movement led by two unique signaling pathways have been demonstrated in individual tumor cells $[6,7]$. Mesenchymal-type movement is characterized by an elongated cellular morphology that results from Rac-dependent actin assembly at the leading edge and requires extracellular proteolysis for Rac-dependent actin protrusions to be pushed through channels in the extracellular matrix. In contrast, amoeboid movement is characterized by a rounded, blebbing morphology that is independent of extracellular proteases [8-10]. These two signaling pathways coordinate to regulate the cytoskeletal rearrangement that plays a critical role in the movement mode transition.

CD147, which belongs to the immunoglobulin superfamily, is a glycosylated transmembrane protein that is highly expressed in various cancers $[11,12]$. Overexpression of CD147 can enhance the metastatic potential of cancer cells due to increased adhesion, migration, invasion, and matrix metalloproteinases 
(MMPs) secretion [13-15]. Our previous study indicated that CD147 inhibits Rho signaling pathways and amoeboid movement by inhibiting Annexin II phosphorylation [16].

The non-receptor tyrosine kinase $\mathrm{Src}$ is overexpressed and activated in many human malignancies $[17,18]$. This kinase associates with many crucial aspects of tumor development and regulates a number of signaling pathways that are involved in survival, adhesion, invasion, migration, and proliferation [1921]. In addition, deregulation of Src leads to constitutive kinase activation and cellular transformation. Decades of research have revealed the crucial role of $\mathrm{Src}$ in regulating small GTPases, and one of the major functions of Src is to modulate the actin cytoskeleton that controls cell migration [22]. However, it remains unknown whether Src is involved in the interconversion between the two modes of individual cell movement and the underlying mechanisms are far from clear.

In the present study, our results demonstrated that CD147 modulates Src activity through FAK when regulating cytoskeletal rearrangement and cell movement. We identified DOCK8 as a GEF for Rac1, which drives mesenchymal-type cell movement. Furthermore, Src promotes STAT3 phosphorylation and STAT3 facilitates DOCK8 transcription, thus enhancing DOCK8 expression and Rac1 activation.

\section{RESULTS}

\section{CD147 promotes mesenchymal-type cell movement}

To better understand the role of CD147 in regulating cell morphology and motility, we established a CD147knockout 7721 cell line (K7721) using the zinc finger nuclease (ZFN)-targeting approach [23]. We first evaluated the expression of CD147 in 7721, K7721 and R7721 cells (Fig. 1A). As shown in Fig. 1B and 1C, cell migration was significantly decreased when CD147 was knocked out, whereas the ability was restored when the expression of CD147 was recovered. We also assessed the invasion ability of the cells, and the results shown in Fig. 1D were consistent with those of the migration assay. Morphological changes were also observed as shown in Fig. 1E; depletion of CD147 produced a more rounded morphology with prominent cortical F-actin in K7721 cells, while R7721 cells displayed a more elongated morphology with bundled F-actin. These results indicated that these changes in cell morphology and motility were due to the loss of CD147.

Mesenchymal-type and amoeboid movements are recognized as interconvertible modes when adapting to different microenvironments and are regulated by the Rac and Rho signaling pathways, respectively. We previously reported that $\mathrm{CD} 147$ promotes the cytoskeletal rearrangement and cell motility in HCC cells. Here, we examined the molecules related with mesenchymal-type and amoeboid movements and found that Rac1-GTP and WAVE2 expression were reduced, while RhoA-GTP and MLC2 phosphorylation were increased, following the depletion of CD147 (Fig. 1F). These results proved that CD147 is involved in the interconvertible cell movement. Similar results were obtained when CD147 was silenced in Huh-7 cells (Fig. 1G).

\section{Inhibition of Src leads to cell morphology and motility changes in $\mathrm{HCC}$ cells}

We first evaluated the effects of Src overexpression on cell morphology. A confocal fluorescence microscopy assay showed that overexpression of Src (Fig. 2A) resulted in a more elongated morphology with prominent cortical F-actin expression (Fig. 2B), which is consistent with mesenchymal-type movement. Then we investigated whether Src plays a dominant role in the changes of cell morphology. Results showed that inhibition of Src activity by Src I-1 (Fig. 2C), one of the gold standards for Src kinase inhibition [24], resulted in a more rounded morphology of 7721 cells (Fig. 2D), which is consistent with amoeboid movement. Wound healing and migration assays revealed that the migration ability of 7721 cells treated with Src I-1 was decreased compared to the solvent control group (Fig. 2E, 2F). In addition, the invasion ability was also significantly down-regulated after Src inhibition (Fig. 2G) without obvious alteration in cell proliferation (Fig. 2H). Interestingly, these phenomena were similar to the phenotype observed after inhibiting CD147 expression and Rac1 signaling pathway as shown in Fig. 1. We next evaluated whether Src is involved in coordinating the Rac/Rho signaling pathway in HCC cells. As shown in Fig. 2I, Src I-1 treatment decreased Rac1 activity (GTP Rac1/total Rac1) and WAVE2 expression in 7721 and HepG2 cells, which also substantially increased RhoA activity (GTP RhoA/total RhoA) and MLC2 activity (p-MLC2/MLC2). These results suggested that Src promotes the Rac1 signaling pathway but inhibits the RhoA signaling pathway in cytoskeletal rearrangement and cell movement in HCC cells, a role similar to that of CD147.

\section{Src is required for CD147-regulated cell movement in $\mathrm{HCC}$ cells}

To verify the functional association between CD147 and $\mathrm{Src}$ in cell movement, we transfected K7721 cells with CD147-pcDNA3.1, and the transfected cells were treated with or without the Src inhibitor Src I-1 (Fig. 3A). As expected, the Src inhibitor blocked the CD147-enhanced cell motility (Fig. 3B) and cytoskeleton rearrangement of 
A

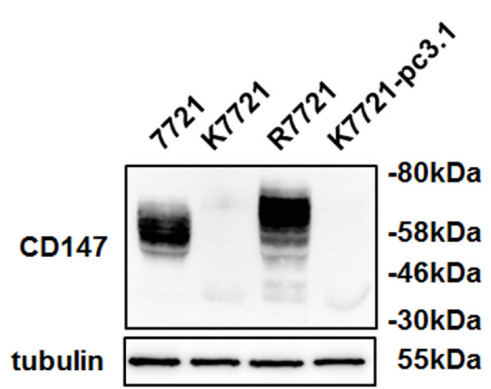

B

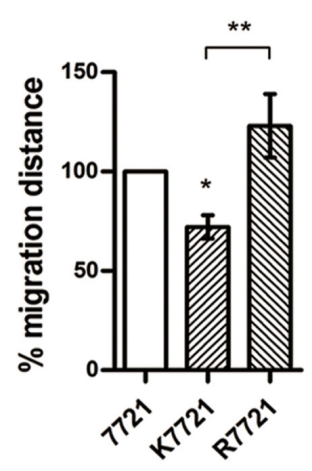

E 7721
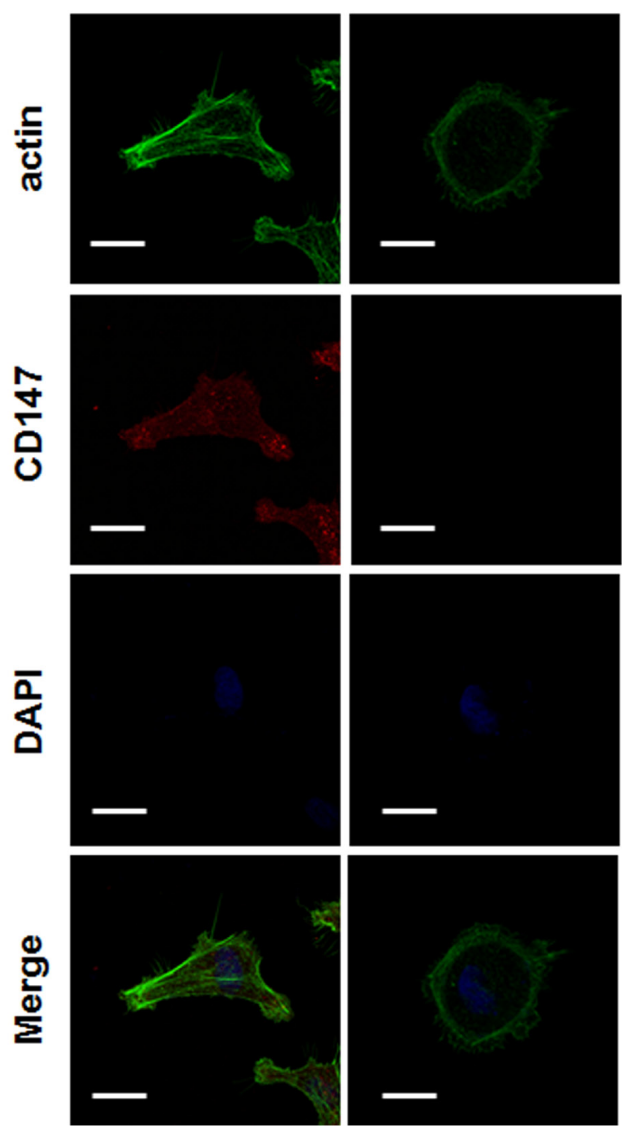

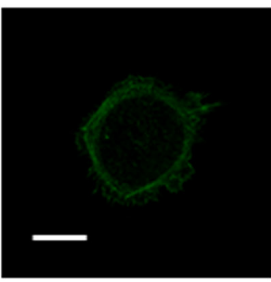

K7721

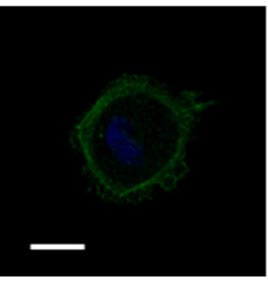

C

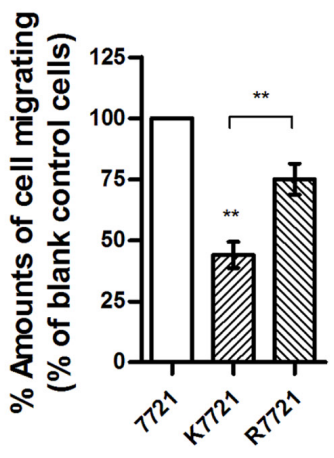

D

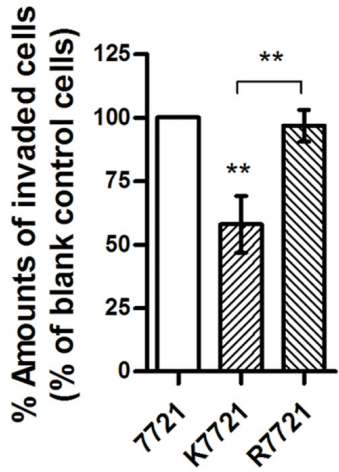

F

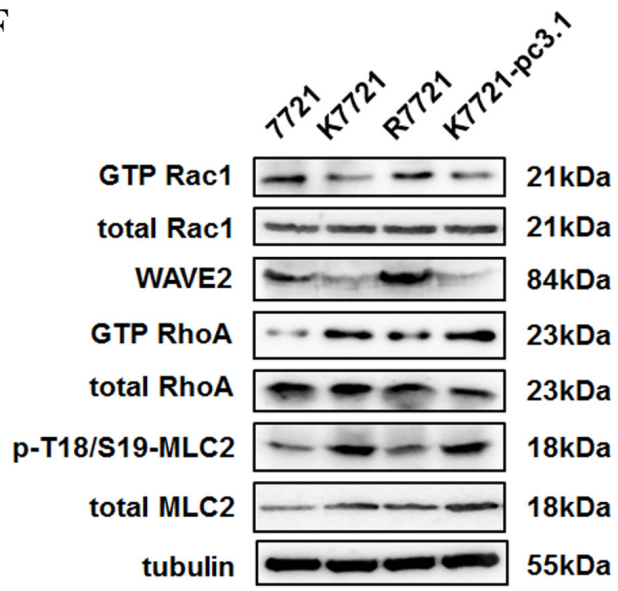

G

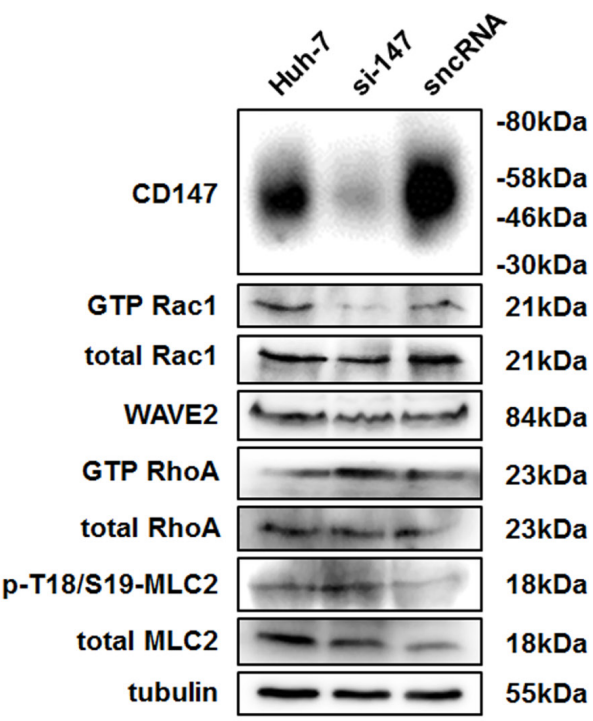

R7721
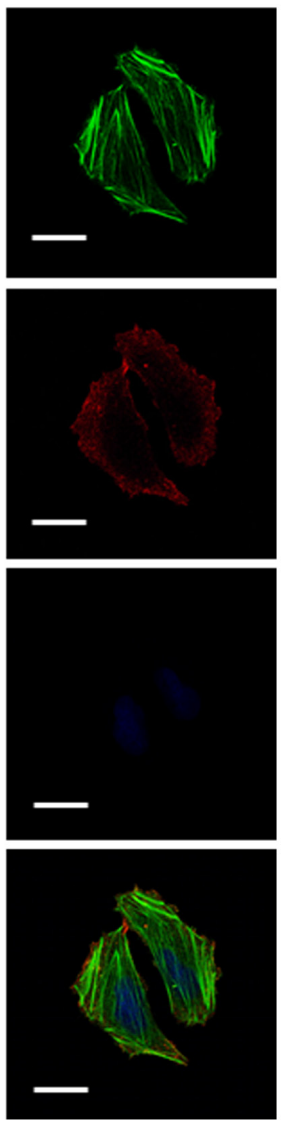

$55 \mathrm{kDa}$

Fig.1: CD147 regulates cell morphology and motility via coordinating Rac and Rho signalings. (A) CD147 was examined in total lysates of 7721, K7721, R7721 and K7721-pcDNA3.1 cells (K7721 cells transfected with pcDNA3.1 vector as a mock control for R7721) using western blotting. (B) Wound healing assay of the 7721, K7721 and R7721 cell lines. (C) Migration assay of the 7721, K7721 and R7721 cell lines. (D) In vitro invasion assay of the 7721, K7721 and R7721 cell lines. (E) Confocal microscopy images of 7721, K7721 and R7721 cells. Red: CD147; Green: actin; Blue: DAPI. Scale bar $=20 \mu \mathrm{m}$. (F) Rac1 activity, WAVE2 expression, and RhoA and MLC2 activities were examined in total lysates of 7721, K7721, R7721 and K7721-pcDNA3.1 cells using western blotting. (G) Src and Rac1 activities, WAVE2 expression, and RhoA and MLC2 activities were examined in total lysates of Huh-7 cells and Huh-7 cells transfected with si-147 or sncRNA using western blotting. The bars represent each sample performed in triplicate, and the error bars indicate \pm SD. $* *$ $\mathrm{P}<0.01, * \mathrm{P}<0.05$, by one-way ANOVA (B-D). 
A

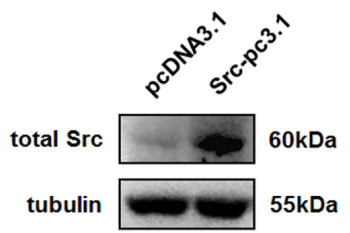

C

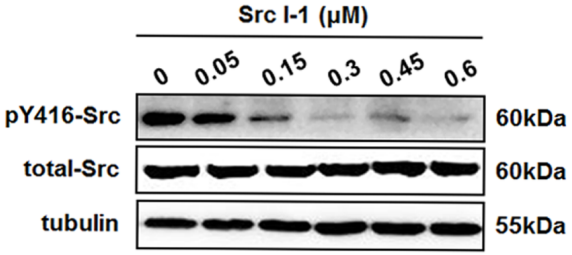

D
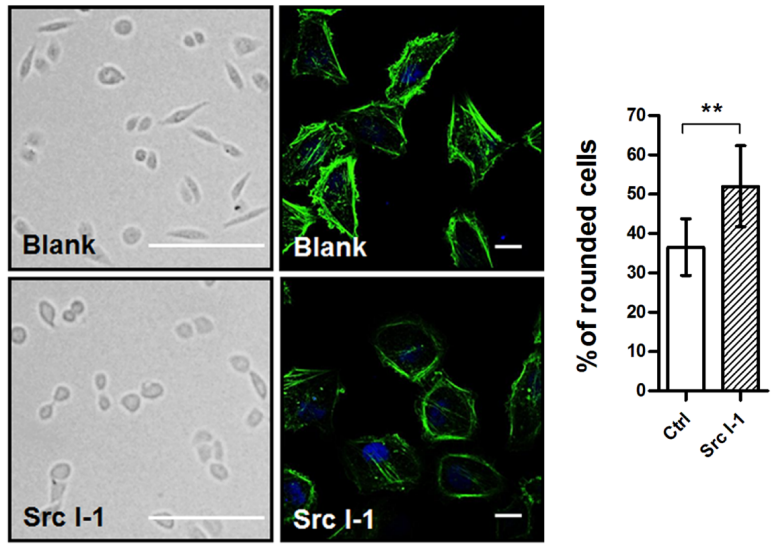

G

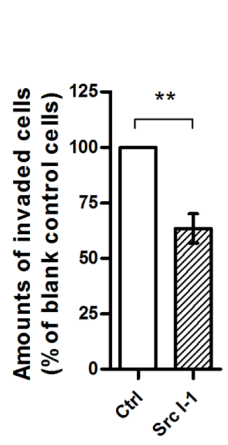

H

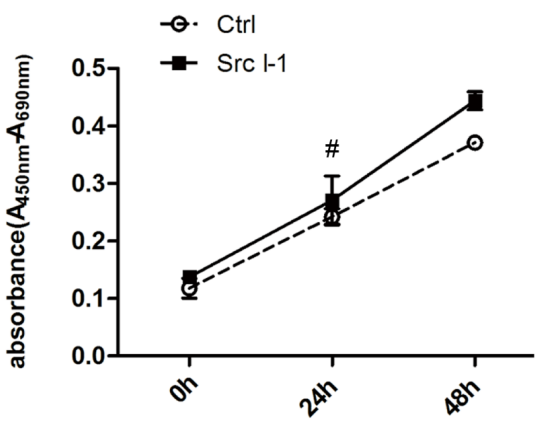

B
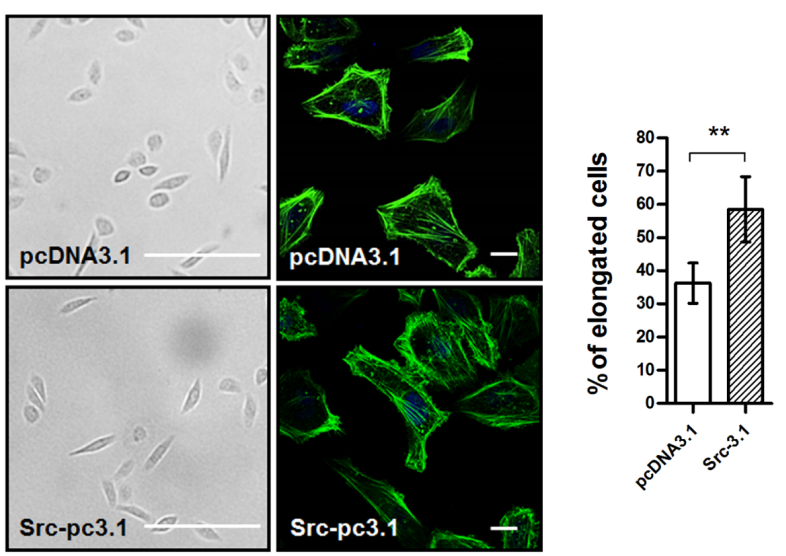

E

F
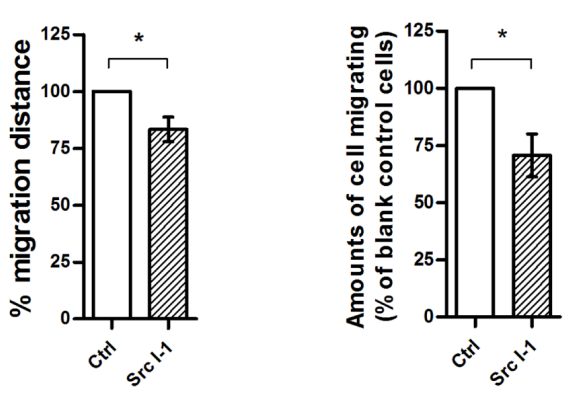

I

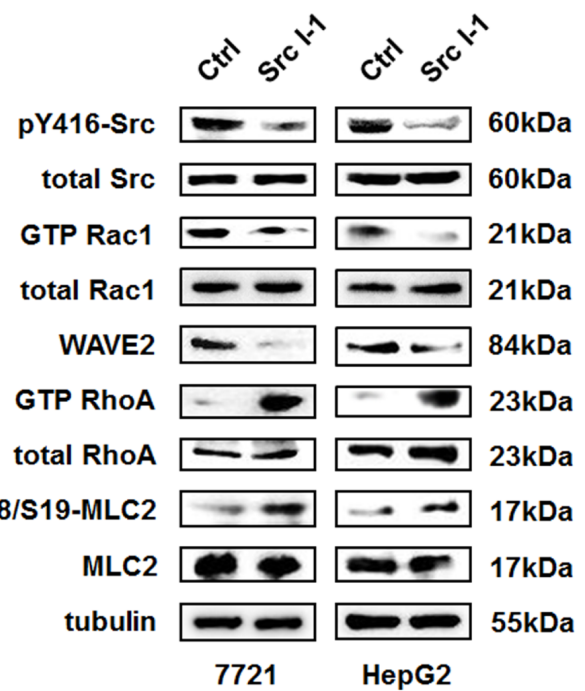

Fig.2: Src activity alteration leads to morphological and migratory activity changes in HCC cells. (A) Src activity level (pY416-Src/total Src) was assessed using western blotting in pcDNA3.1 or Src-pc3.1 transfected 7721 cells. (B) Images (Scale bar = $500 \mu \mathrm{m}$ ) and confocal microscopy images (Scale bar $=20 \mu \mathrm{m}$ ) demonstrating the effect of Src overexpression on morphological changes in 7721 cells. Green: actin; Blue: DAPI. Left panel: representative image. Right panel: quantification. (C) Src activity was assessed using western blotting after treatment of 7721 cells with Src kinase inhibitor (Src I-1). (D) Images (Scale bar $=500 \mu \mathrm{m}$ ) and confocal microscopy images (Scale bar $=20 \mu \mathrm{m})$ demonstrating the effect of Src I-1 treatment on morphological changes in 7721 cells. Green: actin; Blue: DAPI. Left panel: representative image. Right panel: quantification. (E) Effects of Src I-1 treatment on cell motility of 7721 cells. (F) Effects of Src I-1 treatment on cell migration of 7721 cells. (G) Effects of Src I-1 treatment on cell invasion of 7721 cells. (H) Effects of Src I-1 treatment on cell proliferation of 7721 cells. (I) Src and Rac1 activities, WAVE2 expression, and RhoA and MLC2 activities were examined in 7721 and HepG2 cells treated with $300 \mathrm{nM}$ of Src I-1. The bars represent each sample performed in triplicate, and the error bars indicate \pm SD. ** $\mathrm{P}<0.01, * \mathrm{P}<0.05$, \# $\mathrm{P}>0.05$, by t-test $(\mathrm{B}, \mathrm{D}-\mathrm{G})$ and one-way ANOVA $(\mathrm{H})$. 
K7721 cells (Fig. 3C). To confirm the involvement of Src activation in CD147 regulating Rac1 activity, we measured the Rac1-GTP level in CD147-overexpressing cells. As shown in Fig. 3D, CD147 overexpression increased the level of Rac1-GTP, however, Src I-1 treatment abolished the promoting effect of CD147 overexpression on Raclactivity, indicating that CD147 promotes Rac/ WAVE signaling through Src activation. Overall, these data demonstrated that CD147 regulates cell motility by promoting the activation of Src in HCC cells.

\section{CD147 promotes the phosphorylation of Src by FAK}

To investigate the correlation between CD147 and Src, we first evaluated Src activity in 7721 cells

overexpressing CD147. pY416-Src was increased when CD147 was overexpressed in 7721 cells, and this effect was significantly decreased after treatment with Src I-1 (Fig. 4A). Our previous studies demonstrated that CD147 facilitates FAK phosphorylation at Y397 by interacting with $\alpha 3 \beta 1$ integrin [25]. Here, we found that Src activity could be inhibited with the FAK inhibitor and that the enhanced Src activity resulting from CD147 overexpression could be inhibited with either PF573,228 (Fig. 4B) or Y-15 (Fig. 4C). We recapitulated this effect using FAK siRNA (si-FAK) which was designed to knockdown FAK (Fig 4D). These results suggest that CD147 can promote the activation of Src through the integrin/FAK signaling pathway in HCC cells.
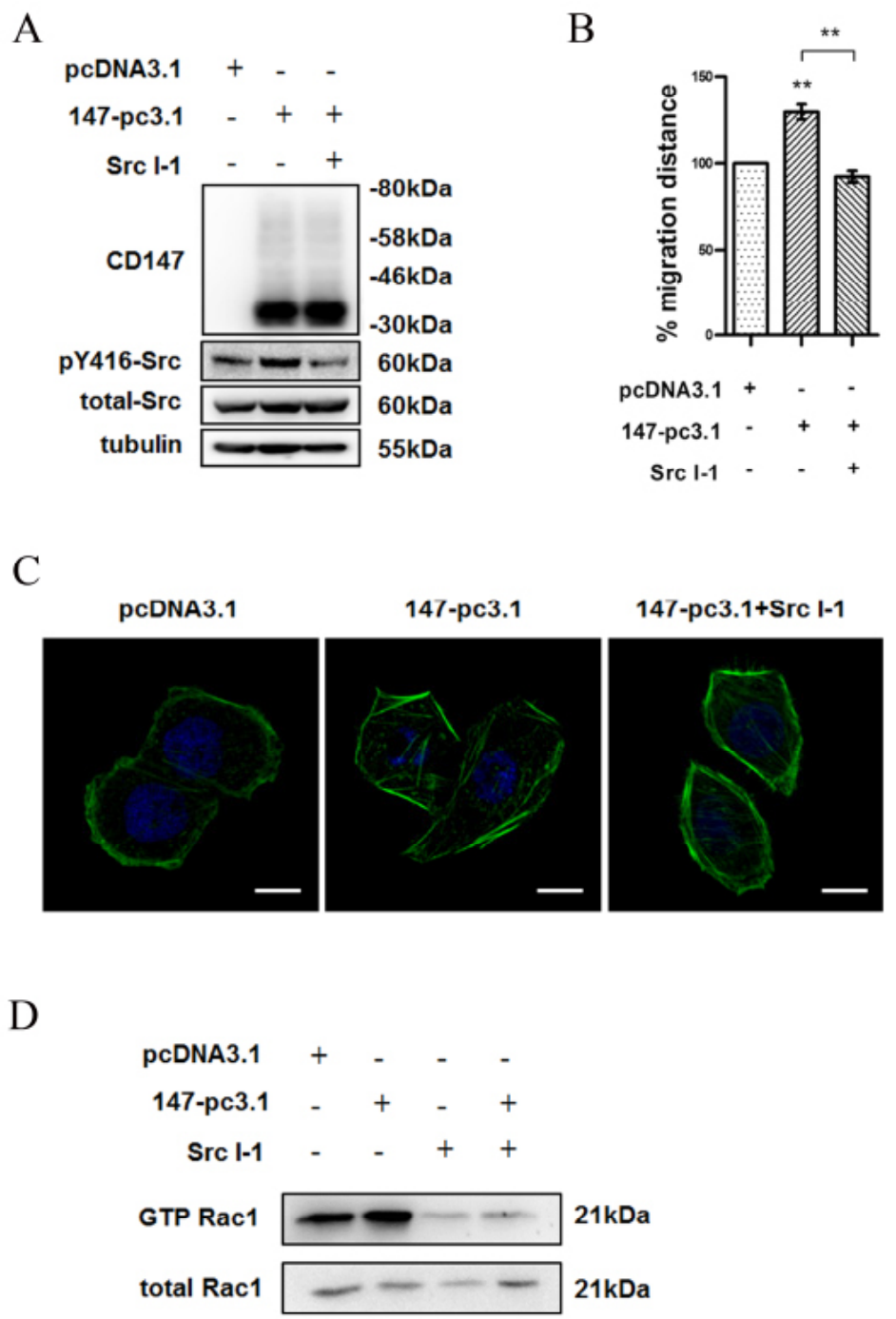

Fig.3: Src is required for CD147 regulated cell movement in HCC cells. (A) Src activity in CD147-pcDNA3.1-expressing K7721 cells treated with Src I-1. (B) Effects of CD147 overexpression and/or Src I-1 treatment on cell motility in 7721 cells. (C) Confocal microscopy images (Scale bar $=10 \mu \mathrm{m}$ ) of CD147 overexpression and/or Src I-1 treatment on cytoskeleton rearrangement in 7721 cells. (D) Rac1 activity in CD147-pcDNA3.1-transfected 7721 cells. The bars represent each sample performed in triplicate, and the error bars indicate $\pm \mathrm{SD}$. ${ }^{* *} \mathrm{P}<0.01$, by one-way ANOVA (B). 
DOCK8 is a Rac1 GEF involved in the mesenchymal movement regulated by CD147 signaling

As neither CD147 nor Src is a GEF or GAP, Rac1 activity cannot be directly regulated by CD147 or Src. To identify the key molecules that directly regulate Rac1 activity in HCC cells, we screened the GEFs in 7721 cells by transfecting these cells with siRNAs targeting GEFs from the DOCK family. Strikingly, most of 7721 cells shifted from an elongated morphology to a rounded morphology (Fig. 5A) following the silencing of DOCK8 (Fig. 5B and 5C), which also led to attenuated cell motility (Fig. 5D). In order to investigate the interaction between Rac1 and DOCK8, we undertook co-immunoprecipitation assays. Protein expression of the two molecules in 7721 and Huh-7 cells was detected by western blotting. As shown in Figure 5E, DOCK8 or Rac1 was then found to co-immunoprecipitate with each other in 7721 and Huh-7 cell lysates, indicating that DOCK8 can physically interact with Rac1 and DOCK 8 is very likely to be a GEF for Rac1 in HCC cells. To confirm this result, we evaluated Rac1 activity and the expression of WAVE2, which is located downstream of Rac1 in the mesenchymal movement signaling pathway. As shown in Fig. 5F, knockdown of DOCK8 resulted in a significant down-regulation of Rac1-GTP and WAVE2 expression, which implied that DOCK8 may regulate Rac1 signaling in HCC cells. Because Rho-ROCK signaling-mediated phosphorylation of MLC2 has been shown to be a key determinant of actomyosin contractility, we also investigated whether DOCK 8 affected the Rho signaling pathway. PhosphoMLC2 (p-T18/S19-MLC2) was significantly increased after knockdown of DOCK8, which implied that DOCK8 promoted Rac1 signaling, but inhibited Rho signaling, in HCC cells. In addition, we previously reported that Annexin II is an activator of Rho signaling [16], we found that the mRNA level of DOCK 8 was significantly up-regulated after Annexin II knockdown in 7721 cells (Fig. 5G). Furthermore, CD147 knockout cells showed substantially reduced DOCK8 expression compared to 7721 or R7721 cells (Fig. 5H). Together, these findings demonstrated that DOCK8 serves as a key Rac1 GEF involved in regulating Rac/Rho signaling, CD147mediated cytoskeletal rearrangement, and cell movement in HCC cells.

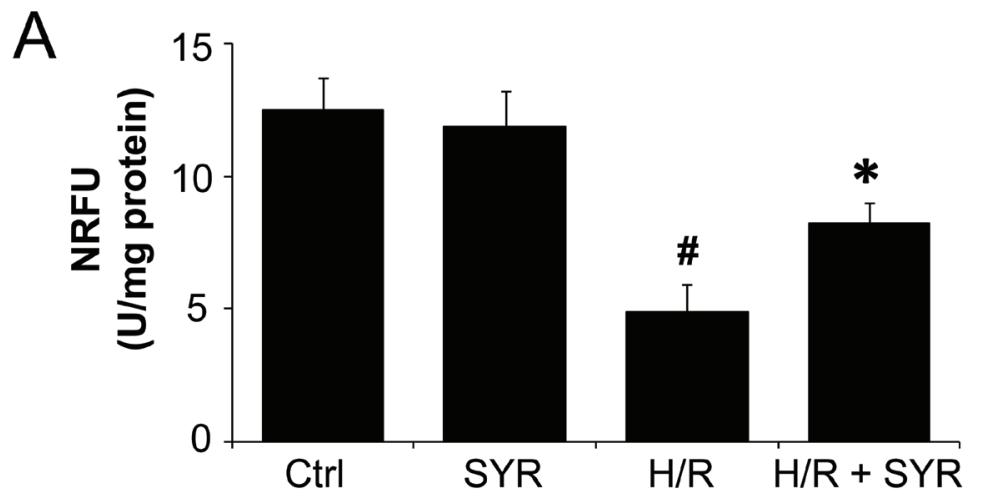

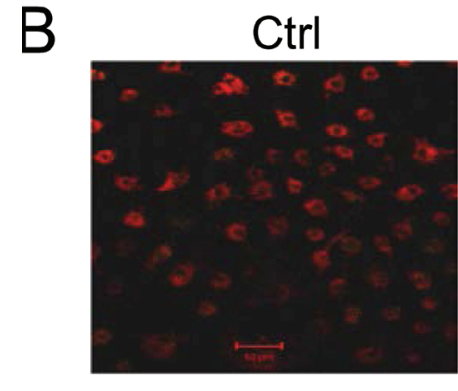

$H / R$

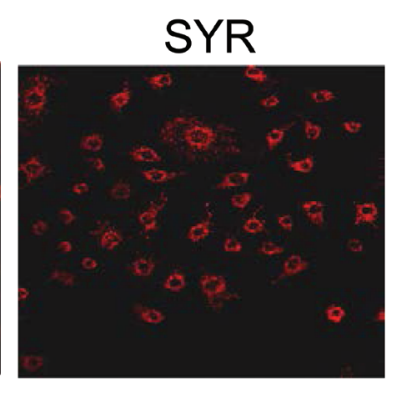

$H / R+S Y R$
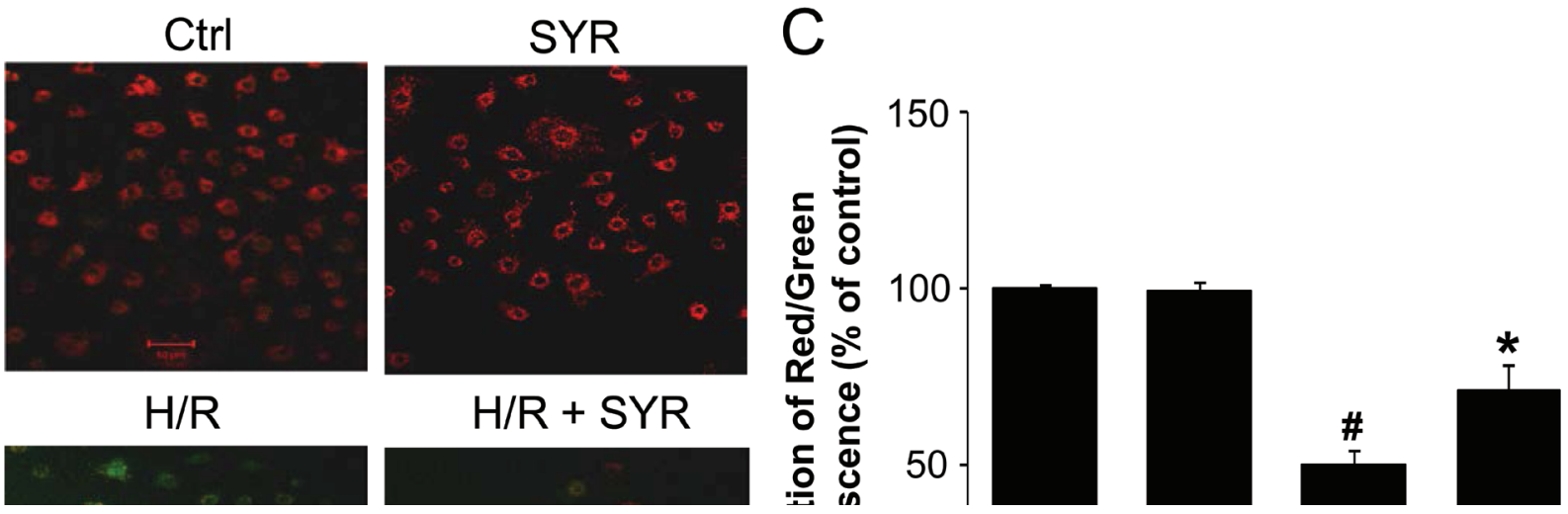

Fig.4: CD147 promotes Src activation through FAK. (A) Src activity in CD147-pcDNA3.1-transfected 7721 cells was blocked with Src I-1. Left panel: representative image. Right panel: quantification. (B) Src activity in 7721 or CD147-pcDNA3.1-transfected 7721 cells was blocked with FAK inhibitor (PF573,228). Left panel: representative image. Right panel: quantification. (C) Src activity in CD147pcDNA3.1-transfected 7721 cells was blocked with FAK inhibitor-14 (Y-15). Left panel: representative image. (D) Src activity in CD147pcDNA3.1-transfected 7721 cells was blocked with FAK siRNA (si-FAK). Left panel: representative image. Right panel: quantification. The bars represent each sample performed in triplicate, and the error bars indicate $\pm \mathrm{SD}$. $* * \mathrm{P}<0.01, * \mathrm{P}<0.05, \# \mathrm{P}>0.05$, by one-way ANOVA. 


\section{Src promotes STAT3 phosphorylation leading to enhanced DOCK8 transcription and expression}

Signal transducer and activator of transcription 3 (STAT3) integrates signals from cytokines and growth factors into transcriptional responses in target cells. It is an important regulator of cancer cell survival and inflammation [26, 27]. And activated STAT3 promotes the transcription of target genes by phosphorylating and translocation from cytoplasm into nucleus. Recent report revealed that $\mathrm{CD} 147$ acts as an upstream activator in STAT3-mediated pancreatic tumor development by forming a signaling complex with CD44s [28]. Katz et al. showed that the invasion of breast cancer is associated
A

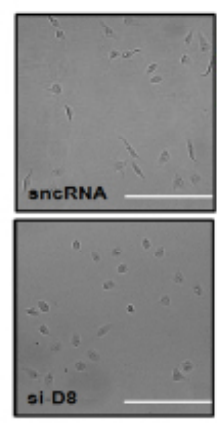

C

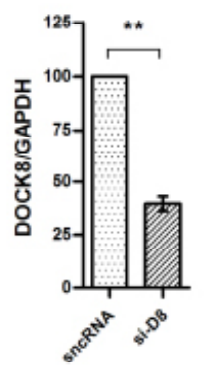

$\mathrm{F}$

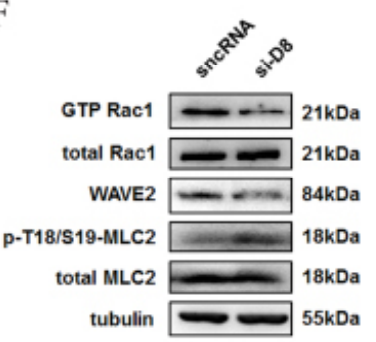

D
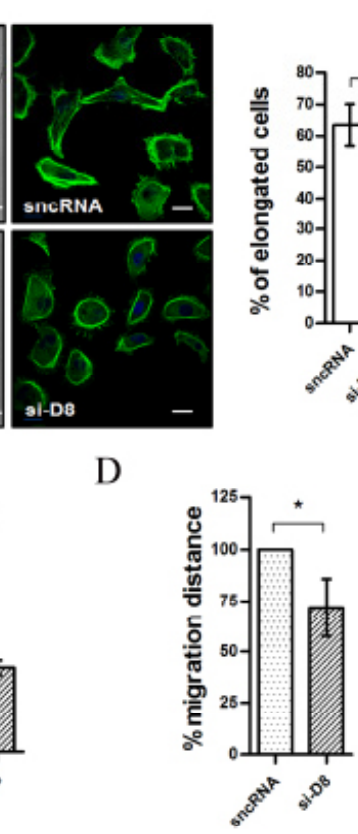

G

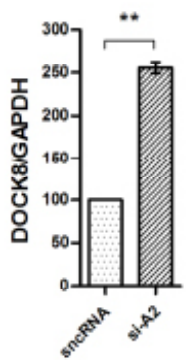

B

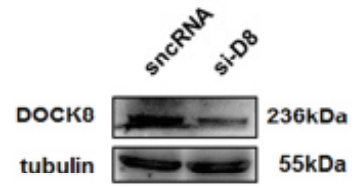

$\mathrm{E}$

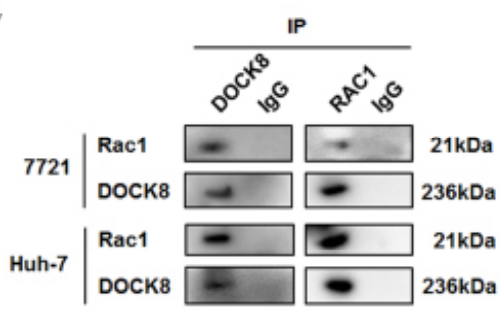

$\mathrm{H}$

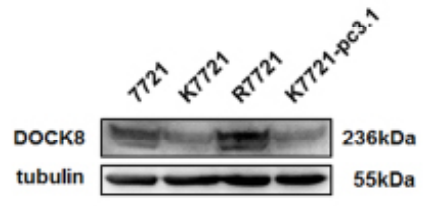

Fig.5: DOCK8 is a Rac1 GEF involved in the Src-regulated Rac/WAVE signaling pathway. (A) Images (Scale bar $=500 \mu \mathrm{m}$ ) and confocal microscopy images (Scale bar $=20 \mu \mathrm{m}$ ) of the DOCK8 siRNA (si-D8)-induced morphological changes in 7721 cells. Green: actin; Blue: DAPI. Left panel: representative image. Right panel: quantification. (B) DOCK8 was detected using western blotting after the transfection of 7721 cells with DOCK8 siRNA. (C) The DOCK8 mRNA level was detected using real-time PCR after the transfection of 7721 cells with si-D8. (D) Effects of si-D8 on cell motility of 7721 cells. Cells were transfected with si-D8 prior to use. (E) Rac1 immunoprecipitated with DOCK8. (F) Rac1 activity, WAVE2 expression and MLC2 activity in 7721 and 7721-siD8 cells. (G) The mRNA level of DOCK8 was detected using real-time PCR after the transfection of 7721 cells with ANXA2 siRNA (si-A2). (H) DOCK8 expression was examined in total lysates of SMMC-7721, K7721, R7721 and K7721-pcDNA3.1 cells using western blotting. The bars represent each sample performed in triplicate, and the error bars indicate $\pm \mathrm{SD}$. ${ }^{*} \mathrm{P}<0.01,{ }^{*} \mathrm{P}<0.05$, by t-test (A, C, D, G). 
with up-regulation of STAT3 and Rac1 activity [29]. We therefore hypothesized that CD147 may promote DOCK8 transcription by enhancing STAT3 phosphorylation. First we explored whether DOCK8 expression is affected by Src activity. As expected, DOCK8 expression in cells treated with Src I-1 was also significantly reduced compared to that in control cells (Fig. 6A). Specific accumulation of DOCK 8 occurred at the leading edge of moving cells, and this accumulation could be attenuated by Src I-1 or CD147 deficiency (Fig. 6B). These results were also observed in HepG2 and Huh-7 cell lines (data not shown). In addition, the decrease of DOCK 8 induced by CD147 deletion could be reversed by expression of Src dominantpositive mutant $\left(\mathrm{Src}_{\mathrm{Y} 530 \mathrm{~F}}\right)$ (Fig. 6C). Then we treated cells with WP1066, a STAT3 pathway inhibitor, results showed that either the protein (Fig. 6D) or the mRNA level of DOCK8 (Fig. 6E) were decreased compared with control groups. Furthermore, the phosphorylation of STAT3 could be inhibited by Src I-1 (Fig. 6F). To determine whether depletion of STAT3 affects CD147-mediated migration, 7721 cells were co-transfected with CD147pcDNA3.1 and STAT3 siRNA (Fig. 6G). As shown in Fig. 6H, STAT3 silencing blocked the CD147-mediated cell motility. Notably, phospho-STAT3 was significantly increased when CD147 was overexpressed. All of these data suggest that CD147 promotes Src-mediated STAT3 phosphorylation, which results in enhanced DOCK8 transcription and expression.

We have shown so far that CD147 promotes HCC cells movement. We investigated whether CD147 could be regulated during $\mathrm{HCC}$ development and progression. We analyzed CD147 mRNA expression utilizing published data from public database Gene Expression Omnibus (GEO). As shown in Fig 6I, increased CD147 expression was found in HCC tissues compared with normal liver tissues. These data suggest that there is an overall upregulation of CD147 expression levels and CD147 plays an important role during HCC progression.

\section{DISCUSSION}

$\mathrm{HCC}$ is a malignant tumor with a high frequency of relapse and metastasis. Moreover, despite recent improvements in long-term survival rates, HCC patient prognosis remains poor [30]. One major hallmark of an aggressive solitary HCC is its ability to metastasize. Thus, understanding the mechanisms that underlie this process may promote the development of effective approaches to reduce $\mathrm{HCC}$ mortality.

Enhanced cell motility is a common feature of tumor metastasis. To migrate, cells undergo dynamic rearrangements of their actin cytoskeleton to form protrusive structures and generate the intracellular forces required for cell translocation. Actin-based cell motility relies on actin as well as numerous actin-interacting proteins and a wide variety of signaling molecules such as kinases and phosphatases that drive the dynamics of the actin system and govern its spatial organization [31].

The Src tyrosine kinase is widely recognized as a key mediator in cytoskeleton organization. Signals initiated by ECM-integrin interactions are transduced into cells through the activation of integrin-associated FAK and Src [32]. Moreover, Src has been shown to be involved in the regulation of Rho-dependent motility and remodeling of the actin cytoskeleton. Recent work from Sebastian et al. Further demonstrates that treatment with the Src inhibitor Dasatinib results in increased RhoA activity and diminished Rac activity in synovial sarcoma cells [33]. Khusial et al. reported that Src stabilizes the expression of Robol by activating the Abl kinase, and increases Rac1 activity to promote tumor cell migration [34]. The results of our present study show that RhoA activity is negatively correlated with the phosphorylation level of Src at Y416. On one hand, Src activity is positively correlated with Rac1 activity and WAVE2 expression, whereas on the other hand, Src plays a negative regulatory role in Rho/ROCK signaling. Enhanced Racl activity promotes mesenchymal-type cell movement and suppresses amoeboid cell movement by maintaining a low phosphorylation level of MLC2, which is consistent with results from previous studies [16].

Accumulating evidence indicates that DOCK family proteins are critical regulators of the small GTPase Rac during several fundamentally important biological processes, such as cell motility and phagocytosis. In mammalian cells, the DOCK family is encoded by at least 11 genes that can be further divided into 4 subgroups. Previous research have identified several novel homologues of DOCK180 and found that many of them directly bind to and exchange GDP for GTP both in vitro and in vivo on either Rac or another Rho-family member, Cdc42. [35]. To explore the mechanisms behind the positive regulation of Rac1 activity, we screened the DOCK180 family of GEFs and identified DOCK8 as a GEF for Rac1 that plays a key role in Src-induced activation of Rac during HCC metastasis. Since the initial report of DOCK8-deficient patients in 2009, DOCK8 has been shown to be essential for the survival of peripheral $\mathrm{T}$ cells and memory CD8 ${ }^{+} \mathrm{T}$ cells [36-38]. DOCK8 is present in lamellipodia and other areas that undergo dynamic actin reorganization [39], and it was also shown that the cellular role of DOCK8 in NK cell-mediated cytotoxicity is achieved, in part, through integrin-mediated adhesion to target cells and by polarization of F-actin and lytic granules at the NK cell cytotoxic synapse [40]. However, the role of DOCK8 in cancer cells remains undefined. Our current study revealed that Src is activated by the CD147-FAK signaling pathway and subsequently upregulates the expression of DOCK8. It has also been reported that the activity of Racl is stimulated by tyrosine phosphorylation of p130Cas, which is an FAK-associated adaptor protein, and that a second adaptor, CRK, can be 
A
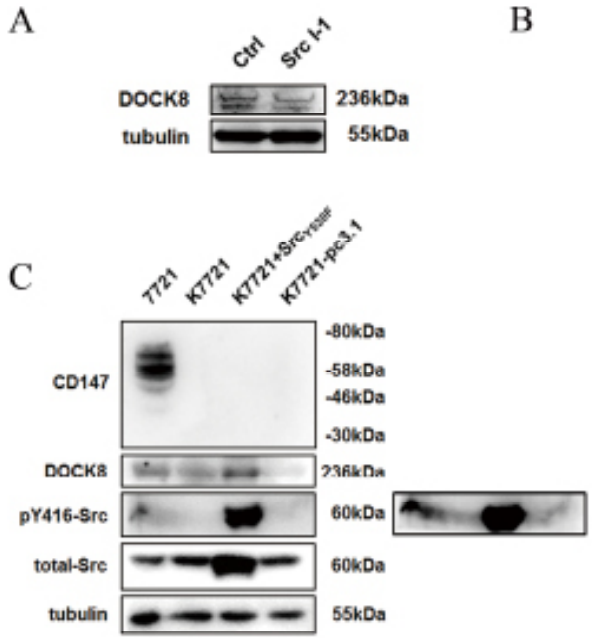

D

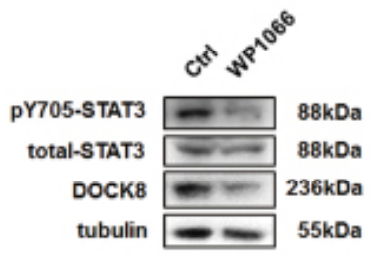

G

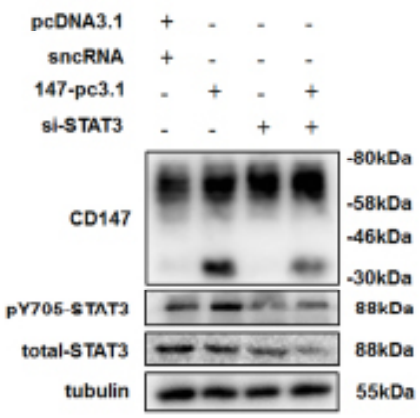

B

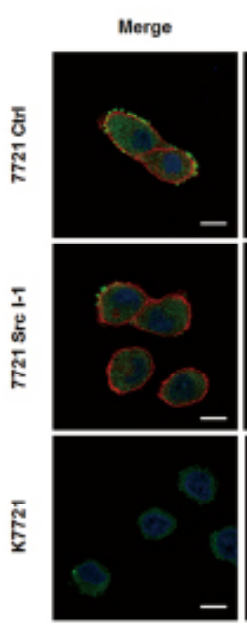

E

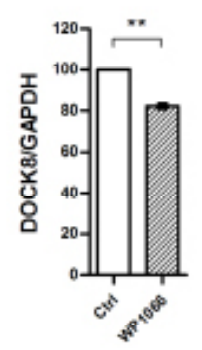

$\mathrm{H}$

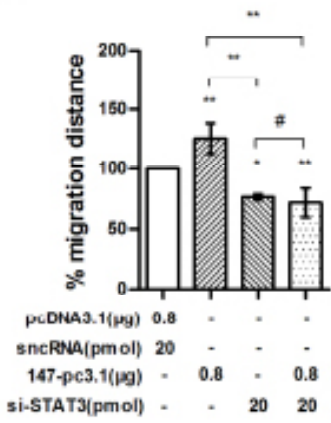

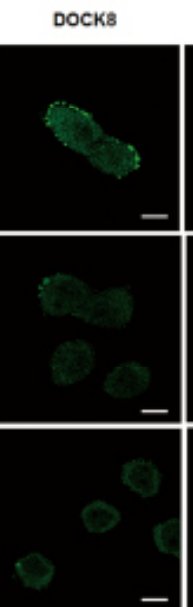
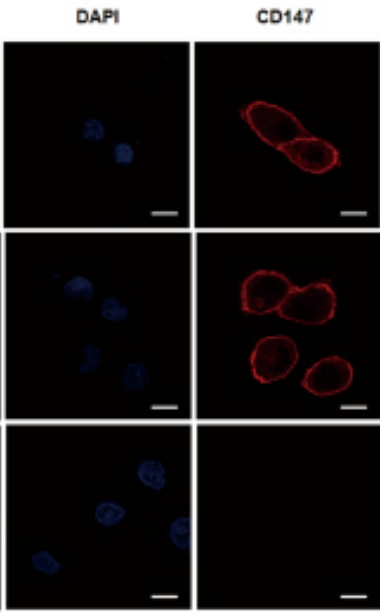

F

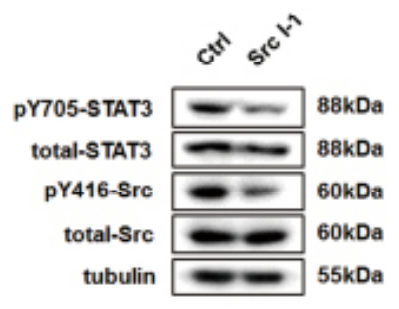

I

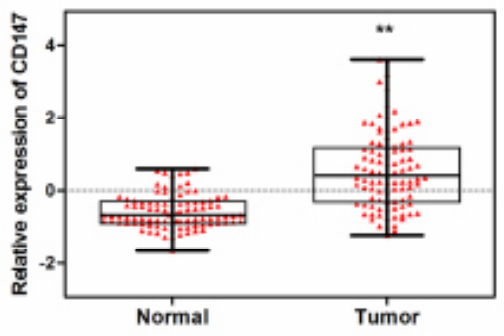

Fig.6: Src promotes DOCK8 expression via enhancing STAT3 phosphorylation. (A) DOCK8 expression was detected using western blotting after treatment of 7721 cells with Src I-1. (B) Confocal microscopy images of 7721, 7721 treated with Src I-1 and K7721 cells. Red: CD147; Green: DOCK8; Blue: DAPI. Scale bar $=20 \mu \mathrm{m}$. (C) DOCK8 expression and Src activity were examined in total lysates of 7721, K7721, K7721 transfected with $\mathrm{Src}_{\mathrm{Y} 530 \mathrm{~F}}$ and K7721-pcDNA3.1 cells (K7721 cells were transfected with pcDNA3.1 vector as a mock control for R7721) using western blotting. Phosphorylation of Src at Tyr416 can also be seen in the overexposed panel (right).(D) Phosphorylation level of STAT3 and DOCK8 expression were detected using western blotting after treatment of 7721 cells with WP1066. (E) The DOCK8 mRNA level was detected using real-time PCR after treatment of 7721 cells with WP1066. (F) Phosphorylation level of STAT3 was detected using western blotting after treatment of 7721 cells with Src I-1. (G) Phosphorylation level of STAT3 and CD147 expression were determined in 7721 cells overexpressing CD147 and/or transfected with STAT3 siRNA. (H) The effects of CD147 overexpression and/or STAT3 silencing on cell motility of 7721 cells. (I) CD147 expression using normalized microarray gene expression data. The bars represent each sample performed in triplicate, and the error bars indicate $\pm \mathrm{SD}$. * $\mathrm{P}<0.05, * * \mathrm{P}<0.01$, \# $\mathrm{P}>0.05$, by unpaired t-test (E), one-way ANOVA $(\mathrm{H})$ and paired t-test (I). 
recruited to phosphorylate p130Cas [41]. Therefore, we hypothesize that the p130Cas-CRK complex brings the CRK-associated Rac GEF DOCK8 to sites of CD147integrin signaling, and DOCK8-activated Rac1 can then stimulate actin polymerization and membrane protrusions, leading to cell motility and invasion. Interestingly, Rac1 inhibition leads to increased Src phosphorylation at Y416, but not at Y527. Conversely, Rac1 activation results in reduced phosphorylation of Src at Y416, but not at Y527 (Supplementary Fig. 1), indicating the existence of a feedback loop between Racl and Src. In fact, we previously demonstrated a positive feedback loop between Rac1 activation and CD147 expression [16]. These feedback loops may help explain the function of CD147 in cytoskeleton reorganization and play an important role in promoting HCC progression.

Previous studies have shown that CD147 interacts with the integrins $\alpha 3 \beta 1$ and $\alpha 6 \beta 1$ in HCC cells and activates the downstream FAK-PI3K- $\mathrm{Ca}^{2+}$ and FAKpaxillin pathways, thus contributing to the processes of cell adhesion, proliferation, differentiation, apoptosis, and tumor progression [42, 43]. Moreover, the interaction of CD147 with the integrin $\beta 1$ subunit can be competitively blocked with the GRGDS peptide, which inhibits downstream FAK signal transduction and actin cytoskeleton rearrangement [25]. Many Rac GEFs have also been reported to be activated by PI3K/PIP3 signaling [44].

As a protease-inducer, CD147 could stimulate the surrounding fibroblasts and endothelial cells to produce matrix metalloproteinases (MMPs) in autocrine and paracrine fashions $[11,15,45,46]$. Recently, multiple studies have provided evidences that CD147 could regulate tumor angiogenesis by stimulating MMPs and VEGF production in tumor and stromal cells [47-50]. Consistent with our findings that CD147 functions in the interconversion between amoeboid and mesenchymal movements in HCC cells (Fig. 7), which is considered a dynamic process in the metastasis of tumor cells, previous work in our lab reported that CD147 promotes the epithelial-mesenchymal transition (EMT) during HCC progression [51]. This finding may provide another piece of evidence supporting the function of CD147 in cytoskeleton rearrangement and mesenchymal movement in HCC cells.

In summary, our studies identify CD147 as a novel regulator of Racl activity that acts through promoting STAT3 phosphorylation and DOCK8 expression, following modulation of Src activation by the integrin/ FAK signaling pathway. Together with our previous results, these suggest that CD147 is an important regulator of the interconversion between amoeboid and

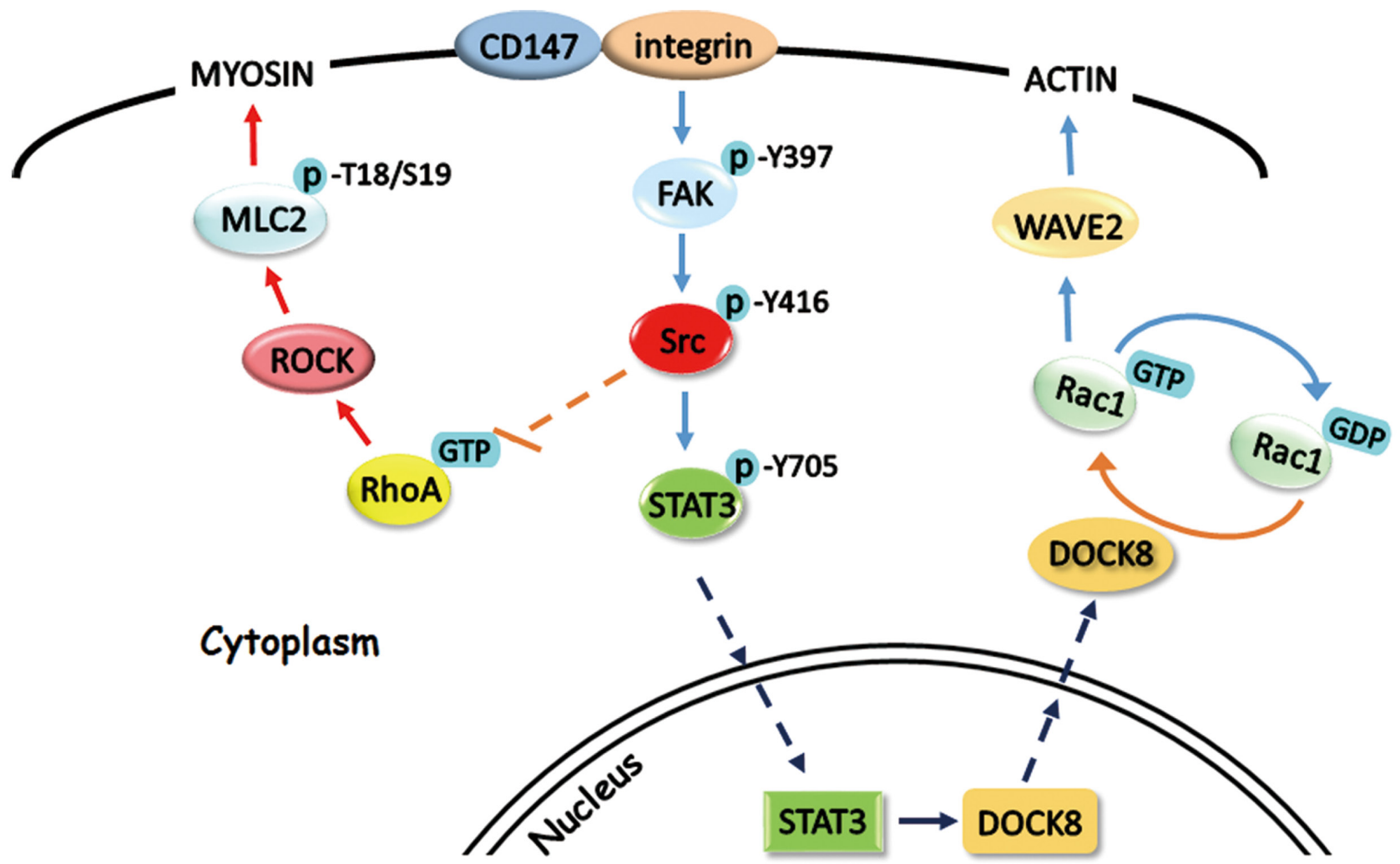

Fig.7: Schematic representation of the major molecular mechanisms of CD147 in regulating hepatocellular carcinoma cells motility. FAK, focal adhesion kinase; DOCK8, dedicator of cytokinesis 8; ROCK, Rho-kinase; MLC2, myosin light chain 2; STAT3, signal transducer and activator of transcription 3. 
mesenchymal movements and is involved in the invasion and metastatic processes of HCC cells. As a novel therapeutic target in the management of $\mathrm{HCC}$, developing drugs that target CD147 may represent a promising strategy for HCC therapy.

\section{MATERIALS AND METHODS}

\section{Cell lines}

Five human HCC cell lines were used: 7721 (SMMC-7721), HepG2, Huh-7, K7721, and R7721. SMMC-7721 and HepG2 cells were purchased from the Institute of Cell Biology, Academic Sinica (Shanghai, China). K7721 cells (7721 CD147 ${ }^{-/}$) and R7721 cells (CD147 is stably expressed in K7721 cells) were developed and preserved in our laboratory. Huh-7 cells were purchased from the Cell Bank of the JCRB (Tokyo, Japan).

\section{Materials}

Antibodies specific for CD147 (sc-9754), $\alpha$-tubulin (sc-8035), WAVE2 (sc-10392), donkey anti-goat IgGFITC (sc-2024) and FAK Inhibitor 14 (Y-15) (sc-203950) were purchased from Santa Cruz (Dallas, Texas); phosphoFAK (Tyr397) (3283), phospho-Src (Tyr416) (2013), phospho-myosin light chain 2 (Thr18/Ser19) (3674), Src (2109), and myosin light chain 2 (3672) antibodies were purchased from Cell Signaling Technology (Boston, MA); DOCK8 (11622-1-AP) and STAT3 (60199-1-Ig) antibodies were obtained from Proteintech (Wuhan, China); phospho-STAT3 (Tyr705) (ab76135) antibody was obtained from Abcam (Cambridge, UK); phospho-Src (Tyr527) (orb14869) antibody was obtained from Biorbyt LLC (San Francisco, CA); and Alexa 594-conjugated goat anti-mouse IgG and Alexa Fluor 488 phalloidin were purchased from Invitrogen (Carlsbad, CA). The Src kinase inhibitor Src I-1, FAK inhibitor PF573,228 (Sigma, St. Louis, MO) and STAT3 inhibitor WP1066 (Merck Millipore, Darmstadt, GER) were also used. Src I-1 was used at $300 \mathrm{nM}$, PF573,228 at $300 \mathrm{nM}, \mathrm{Y}-15$ at $10 \mu \mathrm{M}$ and WP1066 at $5 \mu \mathrm{M}$. The Cell Proliferation Reagent WST-1 was purchased from Roche (Mannheim, GER).

\section{Cell Culture and Plasmids Transfection}

Cells were cultured in RPMI 1640 medium supplemented with $10 \%$ FBS, $1 \%$ penicillin/streptomycin, and $2 \%$ L-glutamine at $37^{\circ} \mathrm{C}$ in a humidified atmosphere of $5 \% \mathrm{CO}_{2}$. Huh-7 cells were cultured in DMEM medium supplemented with $10 \%$ FBS. Plasmids were transfected using Lipofectamine2000 reagent according to the manufacturer's instructions (Invitrogen, Carlsbad, CA).

Full-length CD147 was cloned into pcDNA3.1 (147-pc3.1) as previously prescribed [52]. Fulllength pp60c-Src was cloned into pcDNA3.1 (Srcpc3.1) using primers as follows: forward primer, 5'-AGAATTCATGGGTAGCAACAAGAGCAA

GCCCAAG-3', and reverse primer, 5'-ATACTCGAGCTAGAGGTTCTCCCCGGGCT-3'. To generate $\mathrm{Src}_{\mathrm{Y} 530 \mathrm{~F}}$ with a mutated tyrosine phosphorylation site, primers used were as follows: forward primer, 5'-CGGGCTGGAACTGGGGCTCGGTGG-3', and reverse primer, 5'-CCACCGAGCCCCAGTTCCAGCCCG-3'.

\section{RNA interference}

Chemically synthesized, double-stranded siRNAs were purchased from Shanghai GenePharma Co., Ltd (Shanghai, China). Sequence for CD147 siRNA (si-147) is 5'-GUUCUUCGUGAGUUCCUCtt-3' [11]. siRNA for FAK (sc-35353) was purchased from Santa Cruz (Dallas, Texas), siRNAs for DOCK1 to DOCK11 were designed and synthesized by GenePharma Company. Lipofectamine2000 reagent was employed according to the manufacturer's instructions (Invitrogen, Carlsbad, CA). Silencer negative control siRNA (sncRNA) was used as a negative control.

\section{Western blotting}

HCC cells were harvested in a lysis buffer, the protein quantification was determined by BCA Protein Assay Kit (Thermo Scientific, Waltham, MA) and equal amounts of cellular proteins were subjected to $8 \%-12 \%$ SDS-PAGE separation. Proteins were transferred to polyvinylidene fluoride (PVDF) microporous membranes (Millipore, Boston, MA). Western blotting was performed with the DAB Horseradish Peroxidase Color Development Kit (Beyotime, Shanghai, China) with horseradish peroxidase-conjugated secondary antibodies (Sigma, St. Louis, MO). Tubulin was chosen as an internal control and the blots were probed with mouse $\alpha$-tubulin mAb.

\section{RhoA and Rac1 activity assay}

RhoA and Rac1 activity measurements were carried out according to the Rho (BK036) and Rac (BK035) activation assay kit instructions (Cytoskeleton, Denver, $\mathrm{CO})$.

\section{Immunofluorescence}

Twenty-four hours after siRNA transfection or inhibitor treatment, HCC cells were harvested and allowed 
to attach for $24 \mathrm{~h}$ to Matrigel-pre-coated cell culture dishes with glass bottoms (NEST Biotechnology, Wuxi, China). After washing twice with PBS, the cells were fixed in paraformaldehyde in PBS, permeabilized with $0.1 \%$ Triton X-100, and blocked with $1 \%$ BSA in PBS for $1 \mathrm{~h}$. The dishes were first incubated with the indicated antibodies for $1 \mathrm{~h}$, washed twice with PBS, and then incubated with Alexa 488-phalloidin solution (1:40) and the corresponding FITC-conjugated secondary antibodies for $30 \mathrm{~min}$ in the dark. Cell nuclei were dyed with DAPI (Vector Laboratories, Burlingame, CA). After washing, the dishes were covered with an anti-fade reagent to prevent quenching of the fluorophores, and the cells were visualized using an A1R-A1 confocal laser microscope system (Nikon, Tokyo, Japan).

\section{Real-time RT-PCR analysis}

Total RNA was extracted using an E.Z.N.A.® Total RNA Kit II (Omega Bio-Tek, Norcross, GA) and reversely transcribed into cDNA using a ReverTra Ace-a Kit (Toyobo, Osaka, Japan). SYBR-Green real-time RT-PCR was performed using the Stratagene Mx3005P sequence detection system (Agilent Technologies, Santa Clara, CA) and SYBR Premix EX Taq II (2×) (Takara, Shiga, Japan). GAPDH mRNA was used to normalize the RNA inputs. All primers were synthesized by Shanghai Sangon Biological Engineering Technology \& Services Co., Ltd.

\section{In vitro cell invasion and migration assays}

These assays were performed using chambers containing polycarbonate filters ( $8 \mu \mathrm{m}$ pore size; Merck Millipore, Darmstadt, GER). The upper side of a polycarbonate filter was either coated (invasion) or not coated (migration) with Matrigel (BD Bioscience, San Jose, CA) to form a continuous, thin layer. HCC cells $\left(1 \times 10^{5}\right)$ were re-suspended in $300 \mu \mathrm{L}$ of $0.1 \%$ serum medium with or without Src I-1 and then added to the upper chamber. The lower chamber was filled with $10 \%$ FBS medium $(200 \mu \mathrm{L})$. After a 24 -h (invasion) or 8 -h (migration) incubation and removal of the cells from the upper chamber of the filter with a cotton swab, the cells on the underside were stained and counted.

\section{In vitro wound healing assay}

Twenty-four hours after siRNA transfection or inhibitor treatment, the cells were harvested and seeded in 12-well plates until confluent. Scratching of the monolayer with the tip of a pipette was performed to wound the monolayer. The cells were then washed with serum-free medium and cultured in RPMI 1640 medium with $0.1 \%$ FBS. Photomicrographs at $10 \times$ objective magnification were obtained at various time points $(0 \mathrm{~h}$ and $24 \mathrm{~h})$, and the relative migration distance was calculated using the following formula: the relative migration distance $(\%)=$ 100 (AX-BX) / (A blank-B blank), where $\mathrm{A}$ is the width of the cell wound before incubation, and $B$ is the width of the cell wound after incubation. The results are expressed as the mean $\pm \mathrm{SD}$.

\section{Proliferation assay}

HCC cells $\left(1 \times 10^{3}\right)$ were seeded in 96-well plates and incubated in $100 \mu \mathrm{L}$ serum-free medium 1640. Cells were then treated with or without Src I-1 for $0 \mathrm{~h}, 24 \mathrm{~h}$ and $48 \mathrm{~h}$. Then add $10 \mu \mathrm{L} /$ well WST-1 and incubate for 120 minutes at $37^{\circ} \mathrm{C}$. Plates were then read by a scanning multiwell spectrophotometer by measuring the absorbance of the dye with a wavelength of $450 \mathrm{~nm}$ and a reference wavelength of $690 \mathrm{~nm}$. Same volume of culture medium and WST1 was used as blank control. Three different experiments were performed for each experimental condition.

\section{Co-immunoprecipitation assay}

The interaction of DOCK8 with Rac1 in SMMC7721 and Huh-7 cells was detected using a ProFound Mammalian Co-Immunoprecipitation Kit (Pierce Biotechnology, IL, USA), according to the manufacturer's instructions. Briefly, cells $\left(1 \times 10^{7}\right)$ were lysed using IP Lysis/Wash Buffer. The lysate was collected onto a coupling resin that was pre-bound with $20 \mu \mathrm{g}$ of the mouse anti-human DOCK $8 \mathrm{mAb}$, rabbit anti-human Rac1 $\mathrm{mAb}$ or IgG isotype control, followed by four washes with IP Lysis/Wash Buffer. The coupling resin was then washed with elution buffer, and aliquots of the eluent were analysed by western blotting.

\section{Analysis of CD147 expression from human databases}

Gene expression data of human HCC samples from published microarray study (GEO Accession number GSE22058) was used to analyze the expression of CD147 in HCC progression.

\section{Statistical analysis}

All experiments were performed in triplicate, and the results were expressed as the mean \pm SD. Statistical significance was determined using the GraphPad Prism V5.0 software (GraphPad Software, La Jolla, CA). Differences were deemed significant if $\mathrm{P}<0.05$. Oneway ANOVA was performed for multiple comparisons; two-tailed $t$ test was performed for other experiments to compare the mean values. ** indicates $\mathrm{P}<0.01$, * 
indicates $\mathrm{P}<0.05$, and \# indicates $\mathrm{P}>0.05$. Error bars indicate $\pm \mathrm{SD}$.

\section{ACKNOWLEDGEMENTS}

We thank Gang Nan and Yu-Kui Shang for technical help. This study was supported by grants from the National Natural Science Foundation of China (31371405, 81201774 and 31101005) and the National S\&T Major Project (2012ZX10002017-002, 2013ZX09301301).

\section{Conflict of interest}

The authors declare no conflict of interest.

\section{REFERENCES}

1. Kohn EC. Invasion and metastasis: biology and clinical potential. Pharmacology \& therapeutics. 1991; 52(2):235244.

2. Yilmaz M and Christofori G. Mechanisms of motility in metastasizing cells. Molecular cancer research : MCR. 2010; 8(5):629-642.

3. Price JT and Thompson EW. Mechanisms of tumour invasion and metastasis: emerging targets for therapy. Expert opinion on therapeutic targets. 2002; 6(2):217-233.

4. Kopfstein L and Christofori G. Metastasis: cellautonomous mechanisms versus contributions by the tumor microenvironment. Cellular and molecular life sciences : CMLS. 2006; 63(4):449-468.

5. Vignjevic D and Montagnac G. Reorganisation of the dendritic actin network during cancer cell migration and invasion. Seminars in cancer biology. 2008; 18(1):12-22.

6. Sanz-Moreno V, Gadea G, Ahn J, Paterson H, Marra P, Pinner S, Sahai E and Marshall CJ. Rac activation and inactivation control plasticity of tumor cell movement. Cell. 2008; 135(3):510-523.

7. Parri M and Chiarugi P. Rac and Rho GTPases in cancer cell motility control. Cell communication and signaling : CCS. 2010; 8:23.

8. Yamazaki D, Kurisu S and Takenawa T. Involvement of Rac and Rho signaling in cancer cell motility in 3D substrates. Oncogene. 2009; 28(13):1570-1583.

9. Wolf K, Mazo I, Leung H, Engelke K, von Andrian UH, Deryugina EI, Strongin AY, Brocker EB and Friedl P. Compensation mechanism in tumor cell migration: mesenchymal-amoeboid transition after blocking of pericellular proteolysis. The Journal of cell biology. 2003; 160(2):267-277.

10. Sahai E and Marshall CJ. Differing modes of tumour cell invasion have distinct requirements for Rho/ROCK signalling and extracellular proteolysis. Nature cell biology. 2003; 5(8):711-719.
11. Xu J, Xu HY, Zhang Q, Song F, Jiang JL, Yang XM, Mi L, Wen N, Tian R, Wang L, Yao H, Feng Q, Zhang Y, Xing JL, Zhu P and Chen ZN. HAb18G/CD147 functions in invasion and metastasis of hepatocellular carcinoma. Molecular cancer research : MCR. 2007; 5(6):605-614.

12. Li Y, Xu J, Chen L, Zhong WD, Zhang Z, Mi L, Zhang Y, Liao CG, Bian HJ, Jiang JL, Yang XM, Li XY, Fan CM, Zhu P, Fu L and Chen ZN. HAb18G (CD147), a cancerassociated biomarker and its role in cancer detection. Histopathology. 2009; 54(6):677-687.

13. Jiang JL, Chan HC, Zhou Q, Yu MK, Yao XY, Lam SY, Zhu H, Ho LS, Leung KM and Chen ZN. HAb18G/CD147mediated calcium mobilization and hepatoma metastasis require both $\mathrm{C}$-terminal and $\mathrm{N}$-terminal domains. Cellular and molecular life sciences : CMLS. 2004; 61(16):20832091.

14. Kanekura T, Chen X and Kanzaki T. Basigin (CD147) is expressed on melanoma cells and induces tumor cell invasion by stimulating production of matrix metalloproteinases by fibroblasts. International journal of cancer Journal international du cancer. 2002; 99(4):520528.

15. Sun J and Hemler ME. Regulation of MMP-1 and MMP-2 production through CD147/extracellular matrix metalloproteinase inducer interactions. Cancer research. 2001; 61(5):2276-2281.

16. Zhao P, Zhang W, Wang SJ, Yu XL, Tang J, Huang W, Li Y, Cui HY, Guo YS, Tavernier J, Zhang SH, Jiang JL and Chen ZN. HAb18G/CD147 promotes cell motility by regulating annexin II-activated RhoA and Rac1 signaling pathways in hepatocellular carcinoma cells. Hepatology. 2011; 54(6):2012-2024.

17. Chen ML, Chai CY, Yeh KT, Wang SN, Tsai CJ, Yeh YT and Yang SF. Crosstalk between activated and inactivated c-Src in hepatocellular carcinoma. Disease markers. 2011; 30(6):325-333.

18. Wiener JR, Windham TC, Estrella VC, Parikh NU, Thall PF, Deavers MT, Bast RC, Mills GB and Gallick GE. Activated SRC protein tyrosine kinase is overexpressed in late-stage human ovarian cancers. Gynecologic oncology. 2003; 88(1):73-79.

19. Jin H, Garmy-Susini B, Avraamides CJ, Stoletov K, Klemke RL and Varner JA. A PKA-Csk-pp60Src signaling pathway regulates the switch between endothelial cell invasion and cell-cell adhesion during vascular sprouting. Blood. 2010; 116(25):5773-5783.

20. Boyce B and Xing L. Src inhibitors in the treatment of metastatic bone disease: rationale and clinical data. Clinical investigation. 2011; 1(12):1695-1706.

21. Zhang S and Yu D. Targeting Src family kinases in anticancer therapies: turning promise into triumph. Trends in pharmacological sciences. 2012; 33(3):122-128.

22. Mathew S, George SP, Wang Y, Siddiqui MR, Srinivasan K, Tan L and Khurana S. Potential molecular mechanism 
for c-Src kinase-mediated regulation of intestinal cell migration. The Journal of biological chemistry. 2008; 283(33):22709-22722.

23. Huang Q, Li J, Xing J, Li W, Li H, Ke X, Zhang J, Ren T, Shang Y, Yang H, Jiang J and Chen Z. CD147 promotes reprogramming of glucose metabolism and cell proliferation in HCC cells by inhibiting p53-dependent signaling pathway. J Hepatol. 2014; 61(4): 859-866.

24. Wang L and Damania B. Kaposi's sarcoma-associated herpesvirus confers a survival advantage to endothelial cells. Cancer research. 2008; 68(12):4640-4648.

25. Li Y, Wu J, Song F, Tang J, Wang SJ, Yu XL, Chen $\mathrm{ZN}$ and Jiang JL. Extracellular membrane-proximal domain of HAb18G/CD147 binds to metal ion-dependent adhesion site (MIDAS) motif of integrin beta1 to modulate malignant properties of hepatoma cells. J Biol Chem. 2012; 287(7):4759-4772.

26. Li N, Grivennikov SI and Karin M. The unholy trinity: inflammation, cytokines, and STAT3 shape the cancer microenvironment. Cancer Cell. 2011; 19(4):429-431.

27. Yu H, Pardoll D and Jove R. STATs in cancer inflammation and immunity: a leading role for STAT3. Nat Rev Cancer. 2009; 9(11):798-809.

28. Michels S, Trautmann M, Sievers E, Kindler D, Huss S, Renner M, Friedrichs N, Kirfel J, Steiner S, Endl E, Wurst P, Heukamp L, Penzel R, Larsson O, Kawai A, Tanaka S, et al. SRC signaling is crucial in the growth of synovial sarcoma cells. Cancer research. 2013; 73(8):2518-2528.

29. Katz E, Sims AH, Sproul D, Caldwell H, Dixon MJ, Meehan RR and Harrison DJ. Targeting of Rac GTPases blocks the spread of intact human breast cancer. Oncotarget. 2012; 3(6):608-619.

30. Zhang Y, Hu MY, Wu WZ, Wang ZJ, Zhou K, Zha XL and Liu KD. The membrane-cytoskeleton organizer ezrin is necessary for hepatocellular carcinoma cell growth and invasiveness. Journal of cancer research and clinical oncology. 2006; 132(11):685-697.

31. Qin LX. Inflammatory Immune Responses in Tumor Microenvironment and Metastasis of Hepatocellular Carcinoma. Cancer microenvironment : official journal of the International Cancer Microenvironment Society. 2012.

32. Guo W and Giancotti FG. Integrin signalling during tumour progression. Nature reviews Molecular cell biology. 2004; 5(10):816-826.

33. Michels S, Trautmann M, Sievers E, Kindler D, Huss S, Renner M, Friedrichs N, Kirfel J, Steiner S, Endl E, Wurst P, Heukamp L, Penzel R, Larsson O, Kawai A, Tanaka S, et al. SRC Signaling Is Crucial in the Growth of Synovial Sarcoma Cells. Cancer research. 2013; 73(8):2518-2528.

34. Khusial PR, Vadla B, Krishnan H, Ramlall TF, Shen Y, Ichikawa H, Geng JG and Goldberg GS. Src activates Abl to augment Robol expression in order to promote tumor cell migration. Oncotarget. 2010; 1(3):198-209.

35. Cote JF and Vuori K. Identification of an evolutionarily conserved superfamily of DOCK180-related proteins with guanine nucleotide exchange activity. J Cell Sci. 2002; 115(Pt 24):4901-4913.

36. Jabara HH, McDonald DR, Janssen E, Massaad MJ, Ramesh N, Borzutzky A, Rauter I, Benson H, Schneider L, Baxi S, Recher M, Notarangelo LD, Wakim R, Dbaibo G, Dasouki M, Al-Herz W, et al. DOCK8 functions as an adaptor that links TLR-MyD88 signaling to B cell activation. Nat Immunol. 2012; 13(6):612-620.

37. Randall KL, Chan SS, Ma CS, Fung I, Mei Y, Yabas M, Tan A, Arkwright PD, Al Suwairi W, Lugo Reyes SO, Yamazaki-Nakashimada MA, Garcia-Cruz Mde L, Smart JM, Picard C, Okada S, Jouanguy E, et al. DOCK8 deficiency impairs CD8 T cell survival and function in humans and mice. J Exp Med. 2011; 208(11):2305-2320.

38. Lambe T, Crawford G, Johnson AL, Crockford TL, Bouriez-Jones T, Smyth AM, Pham TH, Zhang Q, Freeman $\mathrm{AF}, \mathrm{Cyster} \mathrm{JG}, \mathrm{Su} \mathrm{HC}$ and Cornall RJ. DOCK8 is essential for T-cell survival and the maintenance of CD8+ T-cell memory. Eur J Immunol. 2011; 41(12):3423-3435.

39. Ruusala A and Aspenstrom P. Isolation and characterisation of DOCK8, a member of the DOCK180-related regulators of cell morphology. FEBS Lett. 2004; 572(1-3):159-166.

40. Shamri R, Young KM and Weller PF. PI3K, ERK, p38 MAPK and integrins regulate CCR3-mediated secretion of mouse and human eosinophil-associated RNases. Allergy. 2013; 68(7):880-889.

41. Nagai Y, Osawa K, Fukushima H, Tamura Y, Aoki K, Ohya K, Yasuda H, Hikiji H, Takahashi M, Seta Y, Seo S, Kurokawa M, Kato S, Honda H, Nakamura I, Maki K, et al. p130Cas plays important roles in osteoclastic bone resorption. J Bone Miner Res. 2013.

42. Tang J, Wu YM, Zhao P, Yang XM, Jiang JL and Chen ZN. Overexpression of HAb18G/CD147 promotes invasion and metastasis via alpha3beta1 integrin mediated FAK-paxillin and FAK-PI3K-Ca2+ pathways. Cellular and molecular life sciences : CMLS. 2008; 65(18):2933-2942.

43. Dai JY, Dou KF, Wang CH, Zhao P, Lau WB, Tao L, $\mathrm{Wu}$ YM, Tang J, Jiang JL and Chen ZN. The interaction of HAb18G/CD147 with integrin alpha6betal and its implications for the invasion potential of human hepatoma cells. BMC cancer. 2009; 9:337.

44. Mertens AE, Roovers RC and Collard JG. Regulation of Tiam1-Rac signalling. FEBS Lett. 2003; 546(1):11-16.

45. Li R, Huang L, Guo H and Toole BP. Basigin (murine EMMPRIN) stimulates matrix metalloproteinase production by fibroblasts. Journal of cellular physiology. 2001; 186(3):371-379.

46. Caudroy S, Polette M, Nawrocki-Raby B, Cao J, Toole BP, Zucker S and Birembaut P. EMMPRIN-mediated MMP regulation in tumor and endothelial cells. Clinical \& experimental metastasis. 2002; 19(8):697-702.

47. Voigt H, Vetter-Kauczok CS, Schrama D, Hofmann UB, Becker JC and Houben R. CD147 impacts angiogenesis 
and metastasis formation. Cancer investigation. 2009; 27(3):329-333.

48. Tang Y, Nakada MT, Rafferty P, Laraio J, McCabe FL, Millar H, Cunningham M, Snyder LA, Bugelski P and Yan L. Regulation of vascular endothelial growth factor expression by EMMPRIN via the PI3K-Akt signaling pathway. Molecular cancer research : MCR. 2006; 4(6):371-377.

49. Dai L, Bratoeva M, Toole BP, Qin Z and Parsons C. KSHV activation of VEGF secretion and invasion for endothelial cells is mediated through viral upregulation of emmprininduced signal transduction. Int J Cancer. 2012; 131(4):834843.

50. Chen Y, Gou X, Ke X, Cui H and Chen Z. Human tumor cells induce angiogenesis through positive feedback between CD147 and insulin-like growth factor-I. PLoS One. 2012; 7(7):e40965.

51. Wu J, Ru NY, Zhang Y, Li Y, Wei D, Ren Z, Huang XF, Chen ZN and Bian H. HAb18G/CD147 promotes epithelialmesenchymal transition through TGF-beta signaling and is transcriptionally regulated by Slug. Oncogene. 2011; 30(43):4410-4427.

52. Jiang JL, Zhou Q, Yu MK, Ho LS, Chen ZN and Chan HC. The involvement of HAb18G/CD147 in regulation of storeoperated calcium entry and metastasis of human hepatoma cells. J Biol Chem. 2001; 276(50):46870-46877. 